\title{
INFLUENCE OF THE GRAIN SIZE AND OF THE ROLLING DEGREE ON THE RECRYSTALLIZATION TEXTURE OF LOW-ALLOYED BRASS
}

\author{
J. SAVOIE, K. LÜCKE \\ Institut für Metallkunde und Metallphysik RWTH Aachen, Germany
}

\section{INTRODUCTION}

The development of microstructure and texture during deformation and annealing of low-alloyed brass has been oft studied and is quite well understood ${ }^{1-3}$. However most investigations on the recrystallization texture development were carried out at high rolling degrees and only few publications exist over a large range of deformation ${ }^{2}$. Furthermore, the influence of initial grain size on the annealing texture were seldom investigated ${ }^{4-6}$, especially using the ODF-analysis ${ }^{5,6}$. In this paper the recrystallization texture of cold rolled $\mathrm{Cu}-2 \% \mathrm{Zn}$ alloy with three different initial grain sizes prior deformation $(15 \mu, 120 \mu$ and $150 \mu)$ are investigated over a large range of deformation ( $40 \%-99 \%$ rolling degrees).

\section{EXPERIMENTAL PROCEDURES}

A Cu-2\% Zn ingot was cast under argon atmosphere from high purity copper and zinc $(>99.995 \%)$. The coarse-grained samples were obtained from the finegrained specimens after annealing treatment at elevated temperature. Mean linear intercept values for grain size were $15 \mu, 120 \mu$ and $150 \mu$. These starting samples with different grain sizes were cold rolled to various reductions (40\%-99\%) and subsequently annealed in order to achieve primary recrystallization.

For the determination of textures the ODF's were calculated from four incomplete pole figures $\{111\},\{002\},\{022\}$ and $\{113\}$ using the series expansion method. The ODF's are represented in $\varphi_{2}$-sections $\left(\Delta \varphi_{2}=5^{\circ}\right)$. For a quantitative evaluation of the texture as well as a reduction of ghost effects, a model ODF consisting of a few Gauss type scattering components was fitted with the experimental ODF. Model ODF's were used for the calculation of volume fractions $\left(M_{i}\right)^{7}$. Scanning electron microscopical investigations of shortly annealed specimens were carried out with the help of a special etching ${ }^{8}$ that enables direct orientation measurements.

\section{RESULTS}

Fig. 1 shows examples of the recrystallization texture of $\mathrm{Cu}-2 \% \mathrm{Zn}$ at $85 \%$ reduction for two initial grain sizes $(15 \mu$ and $150 \mu)$. The strongest effect of increasing grain size is an important decrease of the Cube-orientation $\{001\}<100>$ and of the rotated Cube around the rolling direction $\{025\}<100\rangle$ (Cube-RD) and the arising of the Transition component (U) $\{418\}<744\rangle$ at intermediate reductions. Fig. 2 shows the volume fractions of the main recrystallization components for two initial grain sizes $(15 \mu$ and $150 \mu)$ in function of the rolling degree. The recrystallization components are again represented in Fig. 3 in function of the 
grain size $\left(95 \%, 99 \%\right.$ reductions), where values of $1500 \mu$ are taken from Zhang ${ }^{6}$.

For small grained specimens one observes a strong increase of the Cube up to $97 \%$ rolling degree followed by a slight decrease. First generation$\{122\}<212>$ and second generation (sum of $\{447\}<184>,\{148\}<841>$ and $\{148\}<474>$ ) twins (Tw) of the Cube reach a maximum at $85 \%$ reduction and decrease at higher deformations. Also a relative strong Cube-RD is found at high rolling degrees (>95\%). Additionally the BR- (Brass Recrystallization) $\sim\{236\}<385>$ and the $\mathrm{P}-\{011\}<377>$ are observed above $\sim 80 \%$ reduction.

For coarse grained specimens the Cube is strongly suppressed and reaches $10 \%$ volume fraction above $98 \%$ reduction only. Its twins, especially of the second generation are much stronger than the cube itself. On the other hand, the BR- and P-component develop stronger as in the fine grained samples. The Transition (U) component with some of its twins and the Goss (G) $\{011\}<100>$ orientation arise significantly only in coarse grained samples.

\section{DISCUSSION}

The strong presence of the Cube after annealing of rolled fcc metals of medium SFE has been thouroughly investigated ${ }^{1-4}$ according to the oriented nucleation mechanism after the Dillamore and Katoh (D-K) theorie 9 and subsequent growth selection ${ }^{3,10}$. Fig.4a represents a Cube orientated nucleus which spreads in large areas of the rolling structure with different orientations. This shows the strong compromise character of this high symmetrical orientation.

The decrease of the Cube at high reductions in fine grained samples, which is observed in pure copper ", is explained as followed: 1- By the extension of Cube orientated areas in divergent zones along the rolling direction according to the D-K-theorie, the Cube orientated subgrains are decreasing at high reductions as they rotate away. 2- When the sample thickness reached a value below 100 $\mu \mathrm{m}$, a weakening of the Cube was observed in $\mathrm{Al}^{12}$. This was interpreted through a reduction of Cube nuclei with decreasing the specimen thickness. 3- By the extension of deformation inhomogenities in the rolling structure (shear bands and twins), which disturb (shear bands) and destroy (twins) preferentially the Copper(C) orientation $\{112\}<111\rangle$, wherein most Cube oriented nuclei occur ${ }^{10}$.

The reduction of the $\mathrm{C}$-orientation due to mechanical twinning leads to the occurence of the rolling texture transition and thus of an increase of the Brass(B) orientation $\{011\}<211\rangle$ in which the Cube-RD component and not the Cube itself finds best growth possibilities due to the good $40^{\circ}<111>$ orientation relationship between both B- and Cube-RD-orientations. The occurence of the Cube$\mathrm{RD}$ at high deformations seems thus to be controlled by selected nucleation (rotation of the Cube along RD during rolling) and oriented growth ${ }^{13}$.

The observed reduction of the Cube with increasing initial grain size is connected to the occurence of more developed deformation inhomogenities (especially shear bands) in the rolling structure which destroy the Cube orientated zones 4 . Furthermore, numerous shear bands in coarse grained samples act as preferential nucleation sites, that produce more randomly orientated new grains, which suppress the growth of the Cube.

As only a weak Cube arises in coarse grained samples, also other, less favo- 
rable oriented nuclei can find growth possibilities. Furthermore, the large amount of shear bands produces several nuclei that can grow significantly, if they have a good orientation relationship with the deformed matrix. This is particularly the case of the U-orientation which becomes stronger with increasing grain size. Fig.5a and $5 b$ show the U-orientation which originates in or near a shear band. Due to its good $40^{\circ}<111>$ orientation relationship with the Goss- (which is an important shear band orientation ${ }^{14}$ ) and with the $C / S$-orientation $\{538\}<475>$ (in which shear bands mostly occur ${ }^{13,14}$ ), the U-orientation can grow easily in shear bands (Fig.5a,6a) and in the surrounding matrix (Fig.5b,6b). The increase of the Goss-, P- and BR-orientations with increasing grain size can be explained in the same way, since they originate at least partly in shear bands ${ }^{10,13,15}$.

The annealing twins of the Cube in small grained samples (Fig.4b) reach their maximum at $\sim 85 \%$ reduction and then decrease, although the Cube itself in this deformation range always increases. Due to an augmentation of the frequency of Cube nucleus formation with increasing deformation, the size of the new grains decreases. The probability of growth accident ${ }^{16}$, which can be seen as the origin of annealing twins, becomes weaker leading to a reduction of the twin density. On the other hand, due to weak Cube nucleation in coarse grained specimens, the formation of Cube twins is never suppressed.

\section{CONCLUSION}

The stronger presence of shear bands with increasing grain size and thus the greater proportion of randomly oriented new grains lead to the suppression of the Cube and an increase of the background. The recrystallization texture becomes more random and can be explained as a process of growth selection. Since in fcc metals the $40^{\circ}<111>$ orientation relationship is strongly preferred, the rolling texture of the $85 \%$ rolled coarse grained sample was statistically transformed by all possible $40^{\circ}<111>$ rotations and compared with the corresponding annealing texture (Fig.7). The good concordance between the experimental and the calculated ODF's demonstrates the dominance of orientated growth in coarse grained specimens at least at intermediate reductions, while the recrystallization texture of fine grained specimens is more controlled by the nucleation of the Cube.

\section{REFERENCES}

/1/ U. Schmidt, K. Lücke, J. Pospiech; Proc. ICOTOM 4 (1975), p.147

/2/ K.H. Virnich, K. Lücke; Proc. ICOTOM 5 (1978), p.397

/3/ J. Hirsch; Proc. RISO 7 (1986), p.349

/4/ A.A. Ridha, W.B. Hutchinson; Acta metall. 30 (1982), p.1929

15/ T. Özturk, J.S. Kallend, G.S. Davies; Proc. ICOTOM 6 (1981), p.507

16/ X.M. Zhang; Diss. RWTH Aachen (1987)

17/ K. Lücke, J. Pospiech, K.H. Virnich, J. Jura; Acta metall. 29 (1981), p.167

/8/ G.D. Köhlhoff, X. Sun, K. Lücke; Proc. ICOTOM 8 (1987), p.183

19/ I.L. Dillamore, H. Katoh; Met. Science 8 (1974), p.73

/10/ J. Hirsch; Habilitation thesis RWTH Aachen (1988)

/11/ H. Hammelrath; Diss. RWTH Aachen (1990)

/12/ J. Hirsch, T. Rickert, G. Scharf; VAW unpublished reports

/13/ J. Savoie; Diss. RWTH Aachen (1990) (in preparation)

/14/ M.Y. Huh, J. Hirsch, K. Lücke; Proc. ICOTOM 7 (1984), p.601

/15/ J. Huber, M. Hatherly; Met. Science 13 (1979), p.665

/16/ R.L. Fullman, J.C. Fischer; J. Appl. Phys. 22 (1951), p.1350 
a)

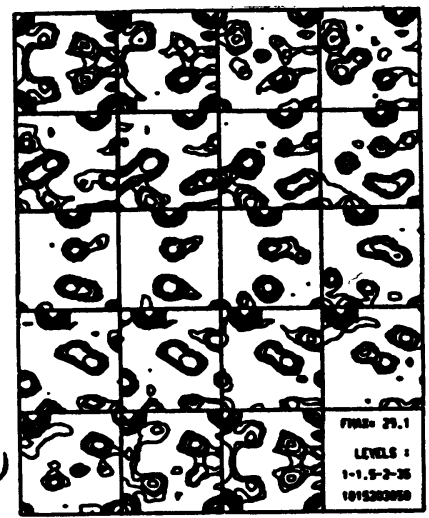

b)

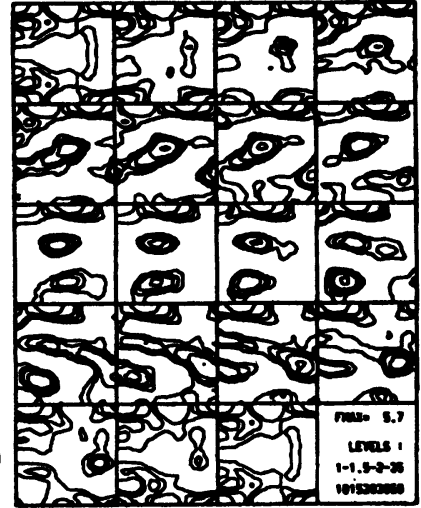

Fig. 1 ODF's of the recrystallization texture of $85 \%$ colled rolled $\mathrm{Cu}-2 \% \mathrm{Zn}$.

(a) fine grained sample $(15 \mu) ;$ (b) coarse grained sample $(150 \mu)$.

a)

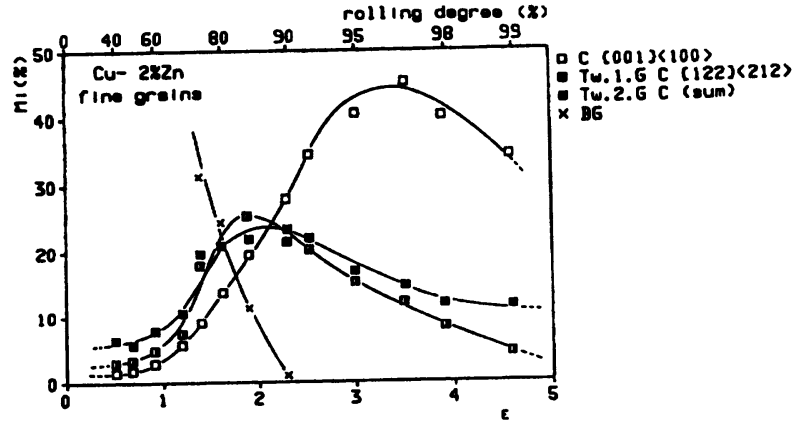

b)

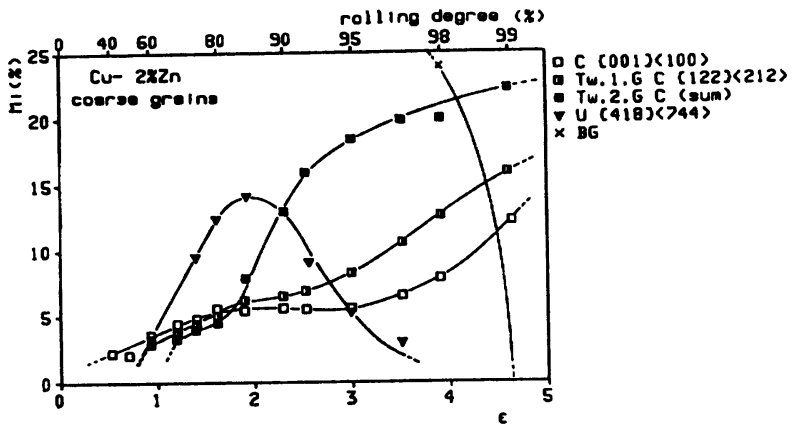

Fig. 2 Volume fractions $M_{1}$ of the main recrystallization components in $\mathrm{Cu}-2 \% \mathrm{Zn}$ as function of the rolling degree.

(a) fine grained samples $(15 \mu)$; (b) coarse grained samples (150 $\mu$ ). 
a)

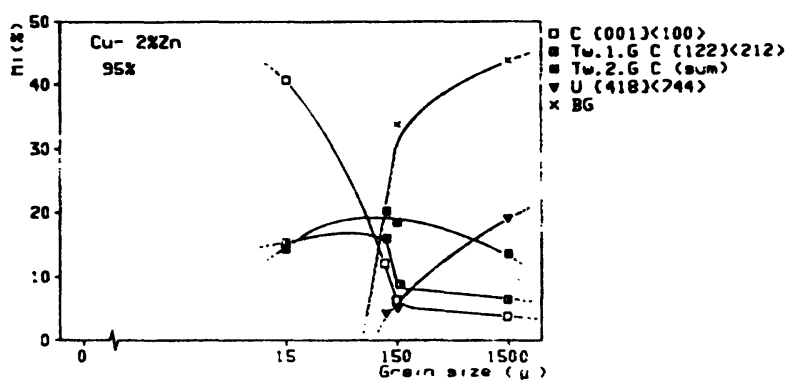

b)

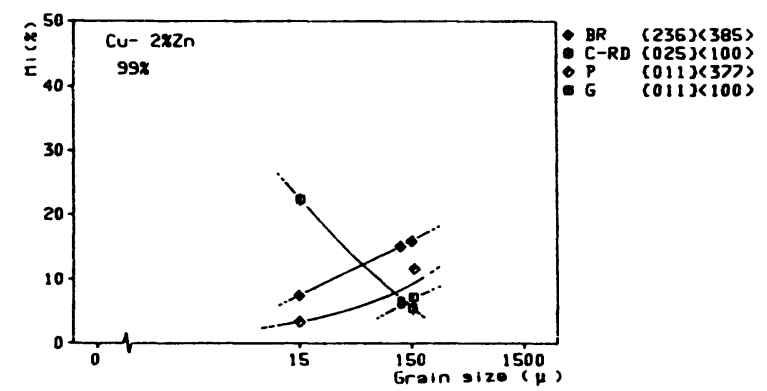

Fig.3 Volume fraction $M_{1}$ of the main recrystallization components in $\mathrm{Cu}-2 \% \mathrm{Zn}$ as function of the grain size at (a) $95 \%$ reduction; (b) $99 \%$ reduction.

a)

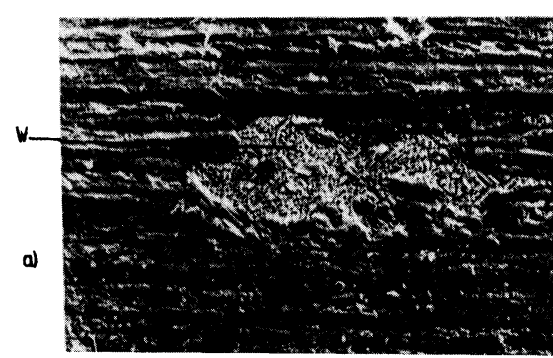

b)

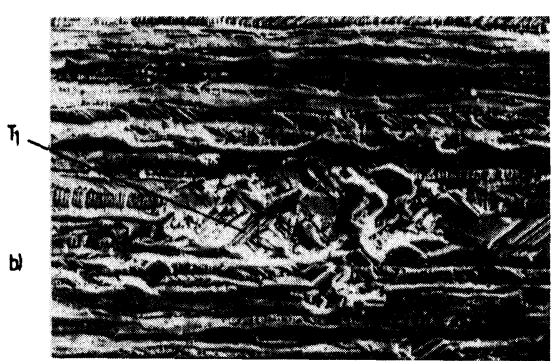

Fig.4 Scanning electron micrograph of partly recrystallized $95 \%$ cold rolled fine grained $\mathrm{Cu}-2 \% \mathrm{Zn}$, showing Cube-oriented grains (W). (x2000) $T_{1}=$ Twin first generation $\{122\}<212>$. 
a)

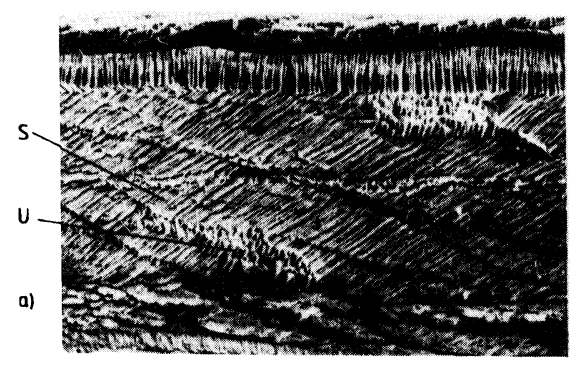

b)

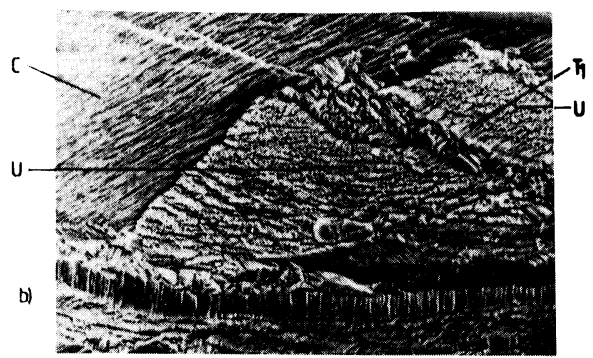

Fig. 5 Scanning electron micrographs of partly recrystallized $85 \%$ cold rolled coarse grained samples showing the Transition-orientation. (x1000)

(a) $\mathrm{Cu}-2 \% \mathrm{Zn}$; (b) $\mathrm{Cu}-5 \% \mathrm{Zn}$. $T_{1}=$ Twin first generation $\{538\}<391>$.

a)

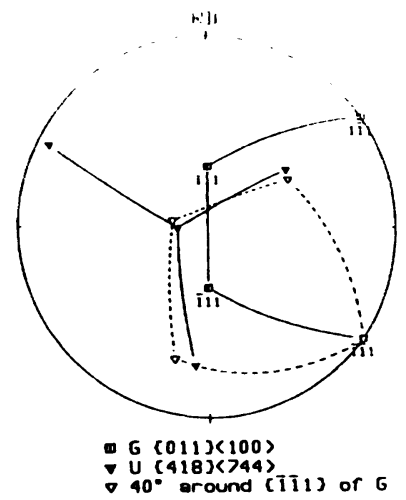

b)

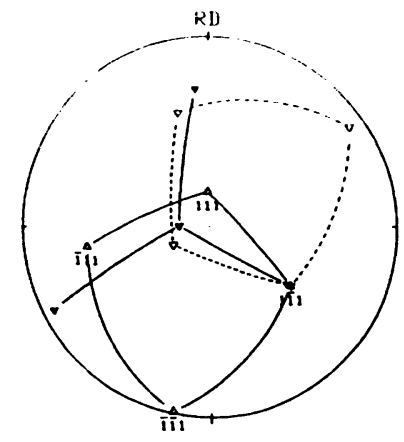

$\Delta \mathrm{C} / \mathrm{S}(538)(475)$
$u$

$\checkmark U$. $(418)\langle>44\rangle)$ around $(111)$ of $\mathrm{C} / \mathrm{S}$

Fig. 6 Orientation relationship between the Transition-orientation $(U)$ and the:

(a) Goss-orientation (G) ; (b) C/S-orientation.

a)

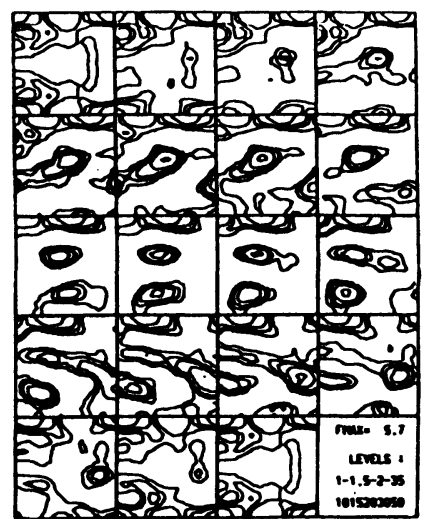

b)

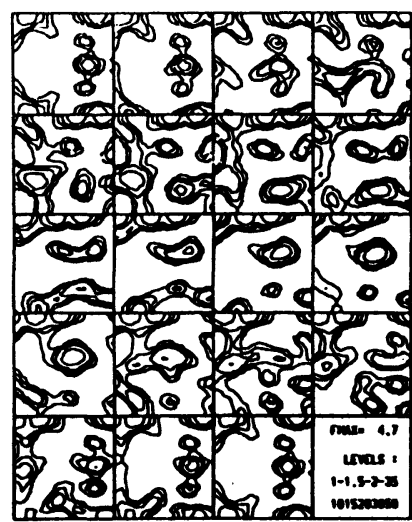

Fig. 7 (a) Recrystallization ODF of the $85 \%$ cold rolled coarse grained $\mathrm{Cu}-2 \% \mathrm{Zn}$;

(b) Transformation $O D F$. 